\title{
The Degree of the Splitting Field of a Random Polynomial over a Finite Field
}

\author{
John D. Dixon and Daniel Panario \\ School of Mathematics and Statistics \\ Carleton University, Ottawa, Canada \\ \{jdixon, daniel\}@math.carleton.ca
}

Submitted: Aug 30, 2004; Accepted: Sep 22, 2004; Published: Sep 30, 2004

Mathematics Subject Classifications: 11T06, 20B99

\begin{abstract}
The asymptotics of the order of a random permutation have been widely studied. P. Erdös and P. Turán proved that asymptotically the distribution of the logarithm of the order of an element in the symmetric group $S_{n}$ is normal with mean $\frac{1}{2}(\log n)^{2}$ and variance $\frac{1}{3}(\log n)^{3}$. More recently R. Stong has shown that the mean of the order is asymptotically $\exp (C \sqrt{n / \log n}+O(\sqrt{n} \log \log n / \log n))$ where $C=2.99047 \ldots$. We prove similar results for the asymptotics of the degree of the splitting field of a random polynomial of degree $n$ over a finite field.
\end{abstract}

\section{Introduction}

We consider the following problem. Let $\mathbb{F}_{q}$ denote a finite field of size $q$ and consider the set $\mathcal{P}_{n}(q)$ of monic polynomials of degree $n$ over $\mathbb{F}_{q}$. What can we say about the degree over $\mathbb{F}_{q}$ of the splitting field of a random polynomial from $\mathcal{P}_{n}(q)$ ? Because we are dealing with finite fields and there is only one field of each size, it is well known that the degree of the splitting field of $f(X) \in \mathcal{P}_{n}(q)$ is the least common multiple of the degrees of the irreducible factors of $f(X)$ over $\mathbb{F}_{q}$. Thus the problem can be rephrased as follows.

Let $\lambda$ be a partition of $n$ (denoted $\lambda \vdash n$ ) and write $\lambda$ in the form $\left[1^{k_{1}} 2^{k_{2}} \ldots n^{k_{n}}\right]$ where $\lambda$ has $k_{s}$ parts of size $s$. We shall say that a polynomial is of shape $\lambda$ if it has $k_{s}$ irreducible factors of degree $s$ for each $s$. Let $w(\lambda, q)$ be the proportion of polynomials in $\mathcal{P}_{n}(q)$ which have shape $\lambda$. If we define $m(\lambda)$ to be the least common multiple of the sizes of the parts of $\lambda$, then the degree of the splitting field over $\mathbb{F}_{q}$ of a polynomial of shape $\lambda$ is $m(\lambda)$. The average degree of a splitting field is given by

$$
E_{n}(q):=\sum_{\lambda \vdash n} w(\lambda, q) m(\lambda) .
$$


An analogous problem arises in the symmetric group $S_{n}$. A permutation in $S_{n}$ is of type $\lambda=\left[1^{k_{1}} 2^{k_{2}} \ldots n^{k_{n}}\right]$ if it has exactly $k_{s}$ cycles of length $s$ for each $s$, and its order is then equal to $m(\lambda)$. If $w(\lambda)$ denotes the proportion of permutations in $S_{n}$ which are of type $\lambda$, then the average order of a permutation in $S_{n}$ is equal to

$$
E_{n}:=\sum_{\lambda \vdash n} w(\lambda) m(\lambda)
$$

We can think of $m(\lambda)$ as a random variable where $\lambda$ ranges over the partitions of $n$ and the probability of $\lambda$ is $w(\lambda, q)$ and $w(\lambda)$ in the respective cases.

Properties of the random variable $m(\lambda)$ (and related random variables) under the distribution $w(\lambda)$ have been studied by a number of authors, notably by Erdös and Turán in a series of papers $[1,2,3]$ and [4]. In particular, the main theorem of [3] shows that in this case the distribution of $\log m(\lambda)$ is approximated by a normal distribution with mean $\frac{1}{2}(\log n)^{2}$ and variance $\frac{1}{3}(\log n)^{3}$ in a precise sense. In our notation the theorem reads as follows. For each real $x$ define

$$
\Psi_{n}(x):=\left\{\lambda \vdash n \mid \log m(\lambda) \leq \frac{1}{2}(\log n)^{2}+\frac{x}{\sqrt{3}}(\log n)^{3 / 2}\right\} .
$$

Then for each $x_{0}>0$ :

$$
\sum_{\lambda \in \Psi_{n}(x)} w(\lambda) \rightarrow \frac{1}{\sqrt{2 \pi}} \int_{-\infty}^{x} e^{-t^{2} / 2} d t \text { as } n \rightarrow \infty \text { uniformly for } x \in\left[-x_{0}, x_{0}\right]
$$

In particular, the mean of the random variable $\log m(\lambda)$ is asymptotic to $\frac{1}{2}(\log n)^{2}$, but this does not imply that $\log E_{n}$ (the $\log$ of the mean of $\left.m(\lambda)\right)$ is asymptotic to $\frac{1}{2}(\log n)^{2}$ and indeed it is much larger. The problem of estimating $E_{n}$ was raised in [4], and the first asymptotic expression for $\log E_{n}$ was obtained by Goh and Schmutz [6]. The result of Goh and Schmutz was refined by Stong [9] who showed that

$$
\log E_{n}=C \sqrt{\frac{n}{\log n}}+O\left(\frac{\sqrt{n} \log \log n}{\log n}\right)
$$

where $C=2.99047 \ldots$ is an explicitly defined constant.

The object of the present paper is to prove analogous theorems for the random variable $m(\lambda)$ under the distribution $w(\lambda, q)$. Actually, it turns out that these theorems hold for several important classes of polynomials which we shall now describe. Consider the classes:

- $\mathcal{M}_{1}(q)$ : the class of all monic polynomials over $\mathbb{F}_{q}$. In this class the number of polynomials of degree $n$ is $q^{n}$ for each $n \geq 1$.

- $\mathcal{M}_{2}(q)$ : the class of all monic square-free polynomials over $\mathbb{F}_{q}$. In this class the number of polynomials of degree $n$ is $\left(1-q^{-1}\right) q^{n}$ for each $n$. 
- $\mathcal{M}_{3}(q)$ : the class of all monic square-free polynomials over $\mathbb{F}_{q}$ whose irreducible factors have distinct degrees. In this class the number of polynomials of degree $n$ is $a(n, q) q^{n}$ where, for each $q, a(n, q) \rightarrow a(q):=\prod_{k \geq 1}\left(1+i_{k}(q) q^{-k}\right) \exp (-1 / k)$ as $n \rightarrow \infty$ where $i_{k}(q)$ is the number of monic irreducible polynomials of degree $k$ over $\mathbb{F}_{q}$ (see [7] Equation (1) with $j=0$ ).

For $x>0$ define

$$
\Phi_{n}(x):=\left\{\lambda \vdash n|| \log m(\lambda)-\frac{1}{2}(\log n)^{2} \mid>\frac{x}{\sqrt{3}}(\log n)^{3 / 2}\right\} .
$$

Then for each of the classes of polynomials described above we have a weak analogue of the theorem of Erdös and Turán quoted above, and an exact analogue of Stong's theorem.

Theorem 1 Fix one of the classes $\mathcal{M}_{i}(q)$ described above. For each $\lambda \vdash n$, let $w(\lambda, q)$ denote the proportion of polynomials in this class whose factorizations have shape $\lambda$. Then there exists a constant $c_{0}>0$ (independent of the class) such that for each $x \geq 1$ there exists $n_{0}(x)$ such that

$$
\sum_{\lambda \in \Phi_{n}(x)} w_{i}(\lambda, q) \leq c_{0} e^{-x / 4} \text { for all } q \text { and all } n \geq n_{0}(x)
$$

In particular, almost all $f(X)$ of degree $n$ in $\mathcal{M}_{i}(q)$ have splitting fields of degree $\exp \left(\left(\frac{1}{2}+\right.\right.$ $\left.o(1))(\log n)^{2}\right)$ over $\mathbb{F}_{q}$ as $n \rightarrow \infty$.

Theorem 2 Let $C$ be the same constant as in the Goh-Schmutz-Stong theorem. Then in each of the classes described above the average degree $E_{n}(q)$ of a splitting field of a polynomial of degree $n$ in that class satisfies

$$
\log E_{n}(q)=C \sqrt{\frac{n}{\log n}}+O\left(\frac{\sqrt{n} \log \log n}{\log n}\right) \text { uniformly in } q .
$$

\section{$2 \quad$ Properties of $w(\lambda, q)$}

First consider the value of $w(\lambda, q)$ for each of the three classes. Let $i_{s}=i_{s}(q)$ denote the number of monic irreducible polynomials of degree $s$ over $\mathbb{F}_{q}$. Then (see, for example, [8]) we have $q^{s}=\sum_{d \mid s} d i_{d}$ so a simple argument shows that

$$
\frac{q^{s}}{s} \geq i_{s} \geq \frac{q^{s}}{s}\left(1+2 q^{-s / 2}\right)^{-1}
$$

Let $\lambda \vdash n$ have the form $\left[1^{k_{1}} \ldots n^{k_{n}}\right]$. Since $\mathcal{P}_{n}(q)$ contains $q^{n}$ polynomials, and there are $\left(\begin{array}{c}i_{s}+k-1 \\ k\end{array}\right)$ ways to select $k$ irreducible factors of degree $s$, we have

$$
w(\lambda, q)=\frac{1}{q^{n}} \prod_{s=1}^{n}\left(\begin{array}{c}
i_{s}+k_{s}-1 \\
k_{s}
\end{array}\right)=\prod_{s=1}^{n} q^{-s k_{s}}\left(\begin{array}{c}
i_{s}+k_{s}-1 \\
k_{s}
\end{array}\right) \text { in } \mathcal{M}_{1}(q) .
$$


Similarly, since there are $\left(1-q^{-1}\right) q^{n}$ polynomials of degree $n$ in $\mathcal{M}_{2}(q)$, and there are $\left(\begin{array}{c}i_{s} \\ k\end{array}\right)$ ways to select $k$ distinct irreducible factors of degree $s$, in this case we have

$$
w(\lambda, q)=\frac{1}{\left(1-q^{-1}\right) q^{n}} \prod_{s=1}^{n}\left(\begin{array}{c}
i_{s} \\
k_{s}
\end{array}\right)=\frac{1}{\left(1-q^{-1}\right)} \prod_{s=1}^{n} q^{-s k_{s}}\left(\begin{array}{c}
i_{s} \\
k_{s}
\end{array}\right) \text { in } \mathcal{M}_{2}(q) .
$$

Finally, since there are $a(n, q) q^{n}$ polynomials of degree $n$ in $\mathcal{M}_{3}(q)$ and each of these polynomials has at most one irreducible factor of each degree, we get

$$
w(\lambda, q)=\frac{1}{a(n, q) q^{n}} \prod_{s=1}^{n}\left(\begin{array}{c}
1 \\
k_{s}
\end{array}\right) i_{s}^{k_{s}}=\frac{1}{a(n, q)} \prod_{s=1}^{n} q^{-s k_{s}}\left(\begin{array}{c}
1 \\
k_{s}
\end{array}\right) i_{s}^{k_{s}} \text { in } \mathcal{M}_{3}(q)
$$

when each part in $\lambda$ has multiplicty $\leq 1$, and $w(\lambda, q)=0$ otherwise. As is well known we also have

$$
w(\lambda)=\frac{1}{1^{k_{1}} 2^{k_{2}} \ldots n^{k_{n}} k_{1} ! k_{2} ! \ldots k_{n} !} .
$$

We shall use the notation $\Pi_{n}$ to denote the set of all partitions of $n, \Pi_{n, k}$ to denote the set of partitions $\left[1^{k_{1}} 2^{k_{2}} \ldots n^{k_{n}}\right]$ in which each $k_{i}<k$ and $\Pi_{n, k}^{\prime}$ to denote the complementary set of partitions.

It is useful to note that in $\mathcal{M}_{1}(q)$ and $\mathcal{M}_{2}(q)$ we have $w(\lambda, q) \rightarrow w(\lambda)$ as $q \rightarrow \infty$. However, this behaviour is not uniform in $\lambda$. Indeed for each of these two classes the ratio $w(\lambda, q) / w(\lambda)$ is unbounded above and below for fixed $q$ if we let $\lambda$ range over all partitions of $n$ and $n \rightarrow \infty$. This means we have to be careful in deducing our theorems from the corresponding results for $w(\lambda)$. In $\mathcal{M}_{3}(q)$, we have $w(\lambda, q)=0$ whenever $\lambda \in \Pi_{n, 2}^{\prime}$, and a simple computation shows that $a(n, q) w(\lambda, q) \rightarrow w(\lambda)$ as $q \rightarrow \infty$ whenever $\lambda \in \Pi_{n, 2}$.

Lemma 3 There exists a constant $a_{0}>0$ such that

$$
1 \leq \frac{1}{1-q^{-1}} \leq a_{0} \text { and } 1 \leq \frac{1}{a(n, q)} \leq a_{0}
$$

for all $n \geq 1$ and all prime powers $q>1$.

Proof. The first inequality is satisfied whenever $a_{0} \geq 2$, so it is enough to prove that the set of all $a(n, q)$ has a strictly positive lower bound.

We shall use results from [7, Theorems 1 and 2]. In our notation [7] shows that $a(q)$ increases monotonically with $q$ starting with $a(2)=0.3967 \ldots$, and that for some absolute constant $c$ we have $|a(n, q)-a(q)| \leq c / n$ for all $n \geq 1$. In particular, $a(n, q) \geq$ $a(q)-c / n \geq a(2)-c / n$. Thus $a(n, q) \geq \frac{1}{2} a(2)>0$ for all $q$ whenever $n>n_{0}:=\lfloor 2 c / a(2)\rfloor$.

On the other hand, as we noted above, in $\mathcal{M}_{3}(q), a(n, q) w(\lambda, q) \rightarrow w(\lambda)$ as $q \rightarrow \infty$ whenever $\lambda \in \Pi_{n, 2}$ and is 0 otherwise. Thus

$$
a(n, q)=\sum_{\lambda \in \Pi_{n}} a(n, q) w(\lambda, q) \rightarrow \sum_{\lambda \in \Pi_{k, 2}} w(\lambda)=b(n), \text { say, as } q \rightarrow \infty
$$


Evidently, $b(n)>0$ (it is the probability that a permutation in $S_{n}$ has all of its cycles of different lengths). Define $b_{0}:=\min \left\{b(n) \mid n=1,2, \ldots, n_{0}\right\}$. Then the limit above shows that there exists $q_{0}$ such that $a(n, q) \geq \frac{1}{2} b_{0}$ whenever $n=1,2, \ldots, n_{0}$ and $q>q_{0}$.

Finally, choose $a_{0} \geq 2$ such that $1 / a_{0}$ is bounded above by $\frac{1}{2} a(2), \frac{1}{2} b_{0}$ and all $a(n, q)$ with $n=1,2, \ldots, n_{0}$ and $q \leq q_{0}$. This value of $a_{0}$ satisfies the stated inequalities.

We next examine some properties of the $w(\lambda, q)$ which we shall need later. In what follows, if $\lambda:=\left[1^{k_{1}} \ldots n^{k_{n}}\right] \in \Pi_{n}$ and $\mu:=\left[1^{l_{1}} \ldots m^{l_{m}}\right] \in \Pi_{m}$, then the join $\lambda \vee \mu$ denotes the partition of $m+n$ with $k_{s}+l_{s}$ parts of size $s$. We shall say that $\lambda$ and $\mu$ are disjoint if $k_{s} l_{s}=0$ for each $s$.

Lemma 4 Let $a_{0}$ be a constant satisfying the conditions in Lemma 3. Then for each class $\mathcal{M}_{i}(q)$ we have $w(\lambda \vee \mu, q) \leq a_{0} w(\lambda, q) w(\mu, q)$ for all $\lambda$ and $\mu$. On the other hand, if $\lambda$ and $\mu$ are disjoint, then $w(\lambda \vee \mu, q) \geq a_{0}^{-2} w(\lambda, q) w(\mu, q)$.

We also have $w(\lambda \vee \mu) \leq w(\lambda) w(\mu)$, with equality holding when $\lambda$ and $\mu$ are disjoint.

Proof. First note that in each of the classes, $w(\lambda \vee \mu, q)$ is 0 if either $w(\lambda, q)$ or $w(\mu, q)$ is 0 . Suppose neither of the latter is 0 and put $r:=w(\lambda \vee \mu, q) / w(\lambda, q) w(\mu, q)$.

First consider the class $\mathcal{M}_{1}(q)$. Then $r$ can be written as a product of terms of the form

$$
\left(\begin{array}{c}
i_{s}+k_{s}+l_{s}-1 \\
k_{s}+l_{s}
\end{array}\right) /\left(\begin{array}{c}
i_{s}+k_{s}-1 \\
k_{s}
\end{array}\right)\left(\begin{array}{c}
i_{s}+l_{s}-1 \\
l_{s}
\end{array}\right) .
$$

The numerator of this ratio counts the number of ways of placing $k_{s}+l_{s}$ indistinguishable items in $i_{s}$ distinguishable boxes. The denominator counts the number of ways of doing this when $k_{s}$ of the items are of one type and $l_{s}$ are another, and so is at least as great as the numerator. Hence we conclude that $r \leq 1<a_{0}$ in this case. Moreover, when $\lambda$ and $\mu$ are disjoint then each term is equal to 1 and so $r=1 \geq a_{0}^{-2}$. This proves the claim for the class $\mathcal{M}_{1}(q)$. Taking limits as $q \rightarrow \infty$ also gives a proof of the final statement.

Now consider the class $\mathcal{M}_{2}(q)$. In this case $r /\left(1-q^{-1}\right)$ can be written as a product of terms of the form

$$
\left(\begin{array}{c}
i_{s} \\
k_{s}+l_{s}
\end{array}\right) /\left(\begin{array}{c}
i_{s} \\
k_{s}
\end{array}\right)\left(\begin{array}{l}
i_{s} \\
l_{s}
\end{array}\right)
$$

The numerator counts the number of ways to choose $k_{s}+l_{s}$ out of $i_{s}$ items, whilst the denominator is at least as large as $\left(\begin{array}{c}i_{s} \\ k_{s}\end{array}\right)\left(\begin{array}{c}i_{s}-k_{s} \\ l_{s}\end{array}\right)$ which counts the number of ways to choose $k_{s}+l_{s}$ items when $k_{s}$ are of one type and $l_{s}$ are another type. This shows that each term is at most 1 and so $r \leq\left(1-q^{-1}\right) \leq a_{0}$ as required. Again, in this case, when the partitions are disjoint, each term is equal to 1 and so $r=1-q^{-1} \geq a_{0}^{-2}$. This proves the claim for the class $\mathcal{M}_{2}(q)$, and the proof for the class $\mathcal{M}_{3}(q)$ is similar (in this case $w(\lambda \vee \mu, q)$ is 0 unless $\lambda$ and $\mu$ are disjoint).

Lemma 5 For all partitions of the form $\left[s^{k}\right]$ and all $q$ we have

$$
w\left(\left[s^{k}\right], q\right) \leq a_{0} \frac{k+1}{(2 s)^{k}}
$$

in each of the classes $\mathcal{M}_{i}(q)$. 
Proof. Fix $q$ and $k$ and define

$$
v_{s}:=\frac{q^{-s k}}{k !} \prod_{j=0}^{k}\left(\frac{q^{s}}{s}+j\right) .
$$

Since $i_{s} \leq q^{s} / s$ we have $w\left(\left[s^{k}\right], q\right) \leq a_{0} v_{s}$ for each of the three classes. We also note that

$$
v_{1}=\frac{1}{k !} \prod_{j=0}^{k-1}(1+j / q) \leq \frac{1}{k !} \prod_{j=0}^{k-1}(1+j / 2)=\frac{k+1}{2^{k}} .
$$

Finally since

$$
v_{s+1} / v_{s}=q^{-k} \prod_{j=0}^{k-1} \frac{q^{s+1} /(s+1)+j}{q^{s} / s+j} \leq q^{-k} \prod_{j=0}^{k-1} \frac{q s}{s+1}=\left(\frac{s}{s+1}\right)^{k},
$$

we obtain $w\left(\left[s^{k}\right], q\right) \leq a_{0} v_{s} \leq a_{0} s^{-k} v_{1}$ so the result follows.

Lemma 6 Let $\lambda=\left[1^{k_{1}} \ldots n^{k_{n}}\right]$ be a partition of $n$. The following are true for each of the classes $\mathcal{M}_{i}(q)$.

(a) If each $k_{s} \leq k$ for some fixed integer $k>0$, then $w(\lambda, q) \leq a_{0} e^{2 k(k-1)} w(\lambda)$.

(b) There exists a constant $c_{1}$ such that, if each $k_{s} \leq 1$, then $w(\lambda, q) \geq c_{1} w(\lambda)$.

Proof. (a) For each of the classes we have

$$
w(\lambda, q) \leq a_{0} \prod_{s=1}^{n} \frac{1}{q^{s k_{s}}} \frac{1}{k_{s} !}\left(i_{s}+k_{s}-1\right)^{k_{s}} .
$$

Using the bound $i_{s} \leq q^{s} / s$ we obtain

$$
\begin{aligned}
w(\lambda, q) & \leq a_{0} \prod_{s=1}^{n} \frac{1}{s^{k_{s}} k_{s} !}\left(1+\frac{s\left(k_{s}-1\right)}{q^{s}}\right)^{k_{s}} \\
& \leq a_{0} w(\lambda) \exp \left(\sum_{s=1}^{n} s k_{s}\left(k_{s}-1\right) q^{-s}\right) .
\end{aligned}
$$

Since $\sum_{s=1}^{\infty} s q^{-s} \leq \sum_{s=1}^{\infty} s 2^{-s}=2$, this proves (a).

(b) Similarly, for partitions with no two parts of the same size we have (for any of the classes)

$$
\begin{aligned}
w(\lambda, q) & \geq \prod_{s=1}^{n} \frac{1}{q^{s k_{s}}} i_{s}^{k_{s}} \geq \prod_{s=1}^{n} \frac{1}{s^{k_{s}} k_{s} !}\left(\frac{1}{1+2 q^{-s / 2}}\right)^{k_{s}} \\
& \geq w(\lambda) \exp \left(-2 \sum_{s=1}^{n} k_{s} q^{-s / 2}\right)
\end{aligned}
$$

so the lower bound follows with $c_{1}:=\exp \left(-2 \sum_{s=1}^{\infty} 2^{-s / 2}\right)=0.007999$.

Recall that the set $\Pi_{n, k}$ consists of all partitions of $n$ in which each part has multiplicity $<k$, and $\Pi_{n, k}^{\prime}$ consists of the remaining partitions. 
Lemma 7 For all classes $\mathcal{M}_{i}(q)$, and all $n$ and $q$

$$
\sum_{\lambda \in \Pi_{n, k}^{\prime}} w(\lambda, q) \leq a_{0}^{2} \frac{k+1}{2^{k-1}} \text { whenever } k \geq 2 .
$$

Similarly

$$
\sum_{\lambda \in \Pi_{n, k}^{\prime}} w(\lambda) \leq \frac{k+1}{2^{k-1}} \text { whenever } k \geq 2 .
$$

Proof. Each $\lambda \in \Pi_{n, k}^{\prime}$ can be written in the form $\left[s^{k}\right] \vee \mu$ for some $\mu \vdash n-k s$ in at least one way. Hence using Lemmas 4 and 5 we obtain

$$
\begin{aligned}
\sum_{\lambda \in \Pi_{n, k}^{\prime}} w(\lambda, q) & \leq \sum_{s=1}^{n / k} \sum_{\mu \vdash n-k s} w\left(\left[s^{k}\right] \vee \mu, q\right) \leq a_{0} \sum_{s=1}^{n / k} w\left(\left[s^{k}\right], q\right) \sum_{\mu \vdash n-k s} w(\mu, q) \\
& =a_{0} \sum_{s=1}^{n / k} w\left(\left[s^{k}\right], q\right) \leq a_{0}^{2} \sum_{s=1}^{\infty} \frac{k+1}{(2 s)^{k}} \leq a_{0}^{2} \frac{k+1}{2^{k-1}} .
\end{aligned}
$$

This proves the stated inequality. The corresponding inequality for $w(\lambda)$ is similar.

\section{Proof of Theorem 1}

Since $\Phi_{n}(x)$ and $\Psi_{n}(x) \backslash \Psi_{n}(-x)$ are complementary sets for $x>0$, and the error function is even, the theorem of Erdös and Turán quoted in the Introduction shows that for fixed $x>0$ :

$$
W_{n}(x):=\sum_{\lambda \in \Phi_{n}(x)} w(\lambda) \rightarrow \eta(x) \text { as } n \rightarrow \infty,
$$

where

$$
\eta(x):=\frac{1}{\sqrt{2 \pi}}\left\{\int_{-\infty}^{-x} e^{-t^{2} / 2} d t+\int_{x}^{\infty} e^{-t^{2} / 2} d t\right\}=\frac{2}{\sqrt{2 \pi}} \int_{x}^{\infty} e^{-t^{2} / 2} d t .
$$

A simple integration by parts shows (see, for example, [5, Chap. 7]) that

$$
\eta(x)<\frac{2 e^{-x^{2} / 2}}{\sqrt{2 \pi} x} \text { for } x>0 .
$$

Thus, for $x \geq 1$, there exists $n_{0}(x)>0$ such that $W_{n}(x)<e^{-x^{2} / 2}$ whenever $n>n_{0}(x)$.

Define $\Phi_{n, k}(x):=\Phi_{n}(x) \cap \Pi_{n, k}$ and $\Phi_{n, k}^{\prime}(x):=\Phi_{n}(x) \cap \Pi_{n, k}^{\prime}$. Now using Lemma 6 we have, for each of the classes $\mathcal{M}_{i}(q)$, that

$$
W_{n, k}(x, q):=\sum_{\lambda \in \Phi_{n, k}(x)} w(\lambda, q) \leq a_{0} e^{2 k(k-1)} \sum_{\lambda \in \Phi_{n, k}(x)} w(\lambda) \leq a_{0} e^{2 k(k-1)} W_{n}(x) .
$$


On the other hand Lemma 7 shows that for $k \geq 1$ :

$$
W_{n, k}^{\prime}(x, q):=\sum_{\lambda \in \Phi_{n, k}^{\prime}(x)} w(\lambda, q) \leq \sum_{\lambda \in \Pi_{n, k}^{\prime}(x)} w(\lambda, q) \leq a_{0}^{2} \frac{k+1}{2^{k-1}}<8 a_{0}^{2} e^{-(k+1) / 2} .
$$

Thus for $x \geq 1, k \geq 1$ and $n \geq n_{0}(x)$ we have

$$
\sum_{\lambda \in \Phi_{n}(x)} w(\lambda, q)=W_{n, k}(x, q)+W_{n, k}^{\prime}(x, q)<a_{0} e^{2 k(k-1)} e^{-x^{2} / 2}+8 a_{0}^{2} e^{-(k+1) / 2} .
$$

If $x \geq 2$, then we can choose $k:=\lfloor x / 2\rfloor$ and obtain

$$
e^{2 k(k-1)} e^{-x^{2} / 2}+8 a_{0} e^{-(k+1) / 2}<e^{-x}+8 a_{0} e^{-x / 4}<\left(1+8 a_{0}\right) e^{-x / 4}
$$

uniformly in $x$. Thus taking $c_{0}:=a_{0}\left(1+8 a_{0}\right)$ we obtain (1) for $x \geq 2$. However, by adjusting the value of $c_{0}$ if necessary we can ensure that the inequality (1) is also valid for $x$ with $1 \leq x<2$. Then the inequality is valid for all $x \geq 1$.

Finally, we prove the last assertion of the theorem. Given any $\varepsilon>0$ and $\delta>0$, choose $x \geq 1$ so that $c_{0} e^{-x / 4}<\delta$, and then choose $n_{1} \geq n_{0}(x)$ so that $x<\varepsilon \sqrt{3 \log n_{1}}$. Now (1) shows that for all $n \geq n_{1}$ the proportion of $f(X)$ of degree $n$ in $\mathcal{M}_{i}(q)$ which have splitting fields whose degree lies outside of the interval $\left[\exp \left(\left(\frac{1}{2}-\varepsilon\right)(\log n)^{2}\right), \exp \left(\left(\frac{1}{2}+\varepsilon\right)(\log n)^{2}\right)\right]$ is bounded by $c_{0} e^{-x / 4}<\delta$. This is equivalent to what is stated.

\section{Proof of Theorem 2}

We start by proving an upper bound for $E_{n}(q)$. Define

$$
\tilde{E}_{n}:=\max \left\{E_{m} \mid m=1,2, \ldots, n\right\} .
$$

(It seems likely that $\tilde{E}_{n}=E_{n}$ but we have not been able to prove this.)

Lemma 8 There exists a constant $c_{2}>0$ such that, in each of the classes $\mathcal{M}_{i}(q), E_{n}(q) \leq$ $c_{2} \tilde{E}_{n}$ for all $q$ and all $n$.

Proof. Let $k \geq 2$ be the least integer such that

$$
a_{0}^{2} \sum_{s=1}^{\infty} \frac{(k+1) s}{(2 s)^{k-1}} \leq 1 / 2 .
$$

We shall define $c_{2}:=2 a_{0} e^{2 k(k-1)}$. 
We shall prove the lemma by induction on $n$. Note that $E_{1}(q)=1 \leq c_{2}=c_{2} \tilde{E}_{1}$. Assume $n \geq 2$ and that $E_{m}(q) \leq c_{2} \tilde{E}_{m}$ for all $m<n$. Now Lemma 4 shows that

$$
\begin{aligned}
E_{n, k}^{\prime}(q) & :=\sum_{\lambda \in \Pi_{n, k}^{\prime}} w(\lambda, q) m(\lambda) \leq \sum_{s=1}^{n / k} \sum_{\mu \vdash n-k s} w\left(\left[s^{k}\right] \vee \mu, q\right) m\left(\left[s^{k}\right] \vee \mu\right) \\
& \leq a_{0} \sum_{s=1}^{n / k} s w\left(\left[s^{k}\right], q\right) \sum_{\mu \vdash n-k s} w(\mu, q) m(\mu) \\
& =a_{0} \sum_{s=1}^{n / k} s w\left(\left[s^{k}\right], q\right) E_{n-k s}(q) .
\end{aligned}
$$

Thus using Lemma 5 , the choice of $k$ and the induction hypothesis, we obtain

$$
E_{n, k}^{\prime}(q) \leq a_{0}^{2} \sum_{s=1}^{n / k} \frac{(k+1) s}{(2 s)^{k-1}} c_{2} \tilde{E}_{n-k s} \leq \frac{1}{2} c_{2} \tilde{E}_{n}
$$

because the sequence $\left\{\tilde{E}_{n}\right\}$ is monotonic. On the other hand, Lemma 6 shows

$$
\begin{aligned}
E_{n, k}(q) & :=\sum_{\lambda \in \Pi_{n, k}} w(\lambda, q) m(\lambda) \\
& \leq a_{0} e^{2 k(k-1)} \sum_{\lambda \in \Pi_{n, k}} w(\lambda) m(\lambda) \leq a_{0} e^{2 k(k-1)} E_{n} \leq \frac{1}{2} c_{2} \tilde{E}_{n}
\end{aligned}
$$

by the choice of $c_{2}$. Hence

$$
E_{n}(q)=E_{n, k}(q)+E_{n, k}^{\prime}(q) \leq c_{2} \tilde{E}_{n}
$$

and the induction step is proved.

To complete the proof of the theorem we must prove a lower bound for $E_{n}(q)$. Let $\Lambda_{n}$ denote the set of partitions $\pi$ of the form:

(i) $\pi$ is a partition of some integer $m$ with $n-r<m \leq n$ where $r$ is the smallest prime $>\sqrt{n}$

(ii) the parts of $\pi$ are distinct and each is a multiple of a different prime $>\sqrt{n}$. Note that if the parts of $\pi$ are $k_{1} r_{1}, \ldots, k_{t} r_{t}$ where $r_{1}, \ldots, r_{t}$ are distinct primes $>\sqrt{n}$ then $w(\pi) m(\pi) \geq \prod_{i} r_{i} /\left(k_{i} r_{i}\right)^{1} 1 !=\prod_{i} 1 / k_{i}$.

Consider the partitions of $n$ which can be written in the form $\pi \vee \omega$ where $\pi \in \Lambda_{n}$ and $\omega \in \Pi_{n-|\pi|}$. In Sect. 3 of [9] (see especially the bottom of page 3) Stong notes (in our notation) that since $\pi$ and $\omega$ are disjoint:

$$
\begin{aligned}
E_{n} & \geq \sum_{\pi \in \Lambda_{n}} \sum_{\omega \vdash n-|\pi|} w(\pi \vee \omega) m(\pi \vee \omega) \\
& \geq \sum_{\pi \in \Lambda_{n}} w(\pi) m(\pi) \sum_{\omega \vdash n-|\pi|} w(\omega)=\sum_{\pi \in \Lambda_{n}} w(\pi) m(\pi) .
\end{aligned}
$$


He then proves that the last sum is greater than $E_{n} \exp \left(-O\left(\frac{\sqrt{n} \log \log n}{\log n}\right)\right)$.

Similarly, using Lemma 4 we obtain

$$
\begin{aligned}
E_{n}(q) & \geq \sum_{\pi \in \Lambda_{n}} \sum_{\omega \vdash n-|\pi|} w(\pi \vee \omega, q) m(\pi \vee \omega) \\
& \geq a_{0}^{-2} \sum_{\pi \in \Lambda_{n}} w(\pi, q) m(\pi) \sum_{\omega \vdash n-|\pi|} w(\omega, q)=a_{0}^{-2} \sum_{\pi \in \Lambda_{n}} w(\pi, q) m(\pi) .
\end{aligned}
$$

Since each $\pi \in \Lambda_{n}$ has all its parts of different sizes, Lemma 6 shows that $w(\pi, q) \geq c_{1} w(\pi)$, and so from the result due to Stong quoted above

$$
E_{n}(q) \geq a_{0}^{-2} c_{1} \sum_{\pi \in \Lambda_{n}} w(\pi) m(\pi) \geq E_{n} \exp \left(-O\left(\frac{\sqrt{n} \log \log n}{\log n}\right)\right) .
$$

The lower bound in our theorem now follows from Stong's theorem.

\section{References}

[1] P. Erdös and P. Turán, On some problems of a statistical group theory I, $Z$. Wahrschein. Verw. Gebeite 4 (1965) 175-186.

[2] P. Erdös and P. Turán, On some problems of a statistical group theory II, Acta Math. Acad. Sci. Hungar. 18 (1967) 151-163.

[3] P. Erdös and P. Turán, On some problems of a statistical group theory III, Acta Math. Acad. Sci. Hungar. 18 (1967) 309-320.

[4] P. Erdös and P. Turán, On some problems of a statistical group theory IV, Acta Math. Acad. Sci. Hungar. 19 (1968) 413-435.

[5] W. Feller, "An Introduction to Probability Theory and its Applications", Vol. 1 (3rd. ed.), Wiley, New York, 1968.

[6] W. Goh and E. Schmutz, The expected order of a random permutation, Bull. London Math. Soc. 23 (1991) 34-42.

[7] A. Knopfmacher and R. Warlimont, Distinct degree factorizations for polynomials over a finite field, Trans. Amer. Math. Soc. 347 (1995) 2235-2243.

[8] R. Lidl and H. Niederreiter, "Finite Fields", Cambridge Univ. Press, 1997.

[9] R. Stong, The average order of a permutation, Electronic J. Combinatorics 5 (1998) \#R41. 